\title{
Randić Index of Some Class of Trees with an Algorithm
}

\author{
H. S. Ramane \\ Department of mathematics, \\ Karnatak University, \\ Dharwad, India.
}

\author{
R. B. Jummannaver \\ Department of mathematics, \\ Karnatak University, \\ Dharwad, India.
}

\author{
V. K. Kyalkonda \\ Department of stastistics, \\ Karnatak University, \\ Dharwad, India.
}

\begin{abstract}
The Randic index $R(G)$ of a graph $G$ is defined as the sum of the weights $\left(d_{G}(u) d_{G}(v)\right)^{-1 / 2}$ over all edges $e=u v$ of $G$. In this paper we have obtained the Randić index of some class of trees and of their complements. Also further developed an algorithmic technique to find Randić index of a graph.
\end{abstract}

Keywords: Algorithm, Degree of a vertex, Randić index, Tree.

\section{INTRODUCTION}

Let $G$ be an undirected graph without loops and multiple edges with $n$ vertices and $m$ edges. Let $V(G)=\left\{v_{1}, v_{2}\right.$, $\left.\ldots, v_{n}\right\}$ be the vertex set of $G$ and $E(G)=\left\{e_{1}, e_{2}, \ldots, e_{n}\right\}$ be the edge set of $G$. There are many types of indices, some based on distance of a graph and some other based on degrees of vertices of graphs. In 1975, the Randić index was proposed by the chemist Milan Randić [6] under the name "branching index". The Randic index $R(G)$ of a graph $G$ is defined as the sum of the weights $\left(d_{G}(u) d_{G}(v)\right)^{-1 / 2}$ over all edges $e=u v$ of $G$, where $d_{G}(u)$ is the degree of a vertex $u$ in $G$. That is,

$$
R(G)=\sum_{u v \in E(G)} \frac{1}{\sqrt{d_{G}(u) d_{G}(v)}}
$$

I. Gutman, et., al studied its mathematical properties and summarized in recent books $[1,2]$. The history of this index is described in [7, 8].It has been found that the Randic index correlates well with the harmonic index [4]. The expressions for the harmonic index and Randic index of the generalized transformation graphs and for their complement graphs were obtained in [5].The adjacency matrix of a graph $G$ is the $n \times n$ matrix $A(G)=\left[a_{i j}\right]$, in which $a_{i j}=1$ if $v_{i}$ is adjacent to $v_{j}$ and $a_{i j}$ $=0$, otherwise [3]. The harmonic index of some trees are obtained and an algorithm for the evaluation of the index is developed in [6].
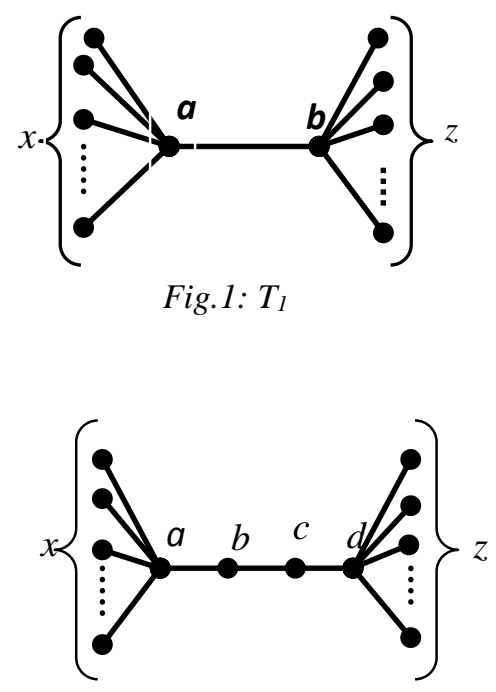

Fig3: $T_{3}$
Fig. 1: $T_{1}$

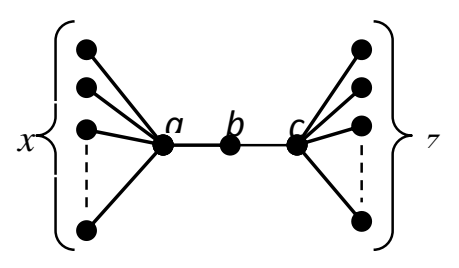

Fig. 2: $T_{2}$

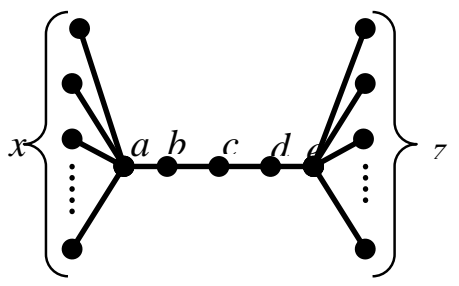

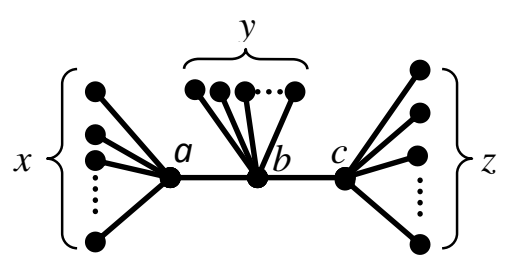

Fig. 5: $T_{5}$

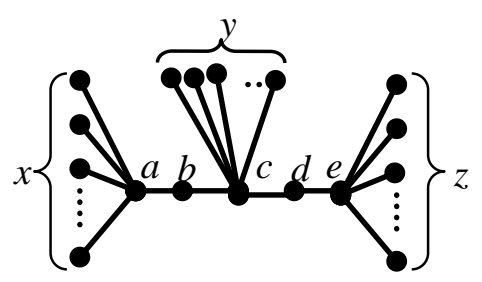

Fig. 7: $T_{7}$

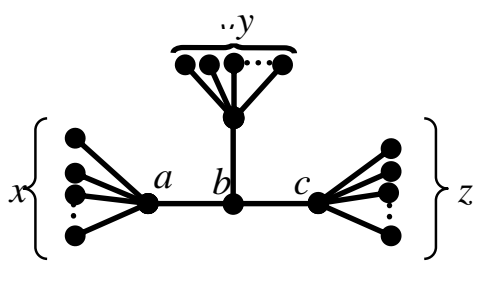

Fig. 6: $T_{6}$

\section{RESULTS:}

Proposition 2.1: If $T_{l}$ is a tree with $n$ vertices as shown in Fig.1, then the Randić index of $T_{l}$ is

$$
R\left(T_{1}\right)=\frac{x}{\sqrt{x+1}}+\frac{n-x-2}{\sqrt{n-x-1}}+\frac{1}{\sqrt{(x+1)(n-x-1)}} .
$$

Proof: Without loss of generality, consider the vertices $a, b$ as shown in Fig. 1, where $d_{T_{1}}(a)=x+1, d_{T_{1}}(b)=n-x-1$. Partition the edge set $E\left(T_{1}\right)$ into 3 sets $E_{1}, E_{2}$ and $E_{3}$ such that $E_{1}=\{u v \mid$ $d_{T_{1}}(u)=1$ and $\left.d_{T_{1}}(v)=x+1\right\}, \quad E_{2}=\left\{u v \mid d_{T_{1}}(u)=1\right.$ and $d_{T_{1}}$ $(v)=z+1\}, E_{3}=\{a b\}$. It is easy to see that $\left|E_{1}\right|=x,\left|E_{2}\right|=n-x-2$, $E_{3} \mid=1$.

Therefore,

$$
R\left(T_{1}\right) \quad=\sum_{u v \in E\left(T_{1}\right)} \frac{1}{\sqrt{d_{T_{1}}(u) d_{T_{1}}(v)}}
$$




$$
\begin{aligned}
& =\sum_{u v \in E_{1}\left(T_{1}\right)} \frac{1}{\sqrt{d_{T_{1}}(u) d_{T_{1}}(v)}}+\sum_{u v \in E_{2}\left(T_{1}\right)} \frac{1}{\sqrt{d_{T_{1}}(u) d_{T_{1}}(v)}}+\sum_{u v \in E_{3}\left(T_{1}\right)} \frac{1}{\sqrt{d_{T_{1}}(u) d_{T_{1}}(v)}} \\
& =\sum_{u v \in E_{1}\left(T_{1}\right)} \frac{1}{\sqrt{(x+1)(1)}}+\sum_{u v \in E_{2}\left(T_{1}\right)} \frac{1}{\sqrt{(n-x-2+1)(1)}}+\sum_{u v \in E_{3}\left(T_{1}\right)} \frac{1}{\sqrt{(x+1)(n-x-2+1)}} \\
& =x \frac{1}{\sqrt{(x+1)}}+(n-x-2) \frac{1}{\sqrt{(n-x-2+1)}}+\frac{1}{\sqrt{(x+1)(n-x-2+1)}} \\
& R\left(T_{1}\right)=\frac{x}{\sqrt{x+1}}+\frac{n-x-2}{\sqrt{n-x-1}}+\frac{1}{\sqrt{(x+1)(n-x-1)}} .
\end{aligned}
$$

The following proposition 2.2 can be proved in analogous to the proposition 2.1 .

Proposition 2.2: If $T_{i}$ is a tree with $n$ vertices as shown in Fig $i$, then the Randić index of $T_{i}$

$i=2,3,4$ as follows,

(i) $R\left(T_{2}\right)=\frac{x}{\sqrt{x+1}}+\frac{n-x-3}{\sqrt{n-x-2}}+\frac{1}{\sqrt{2(x+1)}}+\frac{1}{\sqrt{2(n-x-2)}}$

(ii) $R\left(T_{3}\right)=\frac{1}{2}+\frac{x}{\sqrt{x+1}}+\frac{n-x-4}{\sqrt{n-x-3}}+\frac{1}{\sqrt{2(x+1)}}+\frac{1}{\sqrt{2(n-x-3)}}$

(iii) $R\left(T_{4}\right)=1+\frac{x}{\sqrt{x+1}}+\frac{n-x-5}{\sqrt{n-x-4}}+\frac{1}{\sqrt{2(x+1)}}+\frac{1}{\sqrt{2(n-x-4)}}$

Proposition 2.3: If $T_{5}$ is a tree with $n$ vertices as shown in Fig.5, then the Randic index of $T_{5}$ is

$$
\begin{aligned}
& R\left(T_{5}\right)=\frac{x}{\sqrt{x+1}}+\frac{y}{\sqrt{y+2}}+\frac{n-x-y-3}{\sqrt{n-x-y-2}}+\frac{1}{\sqrt{(x+1)(y+2)}} \\
& +\frac{1}{\sqrt{(y+2)(n-x-y-2)}} .
\end{aligned}
$$

Proof: Without loss of generality consider the vertices $a, b, c$ as shown in Fig. 5, where $d_{T_{5}}(a)=x+1, d_{T_{5}}(b)=y+2, \quad d_{T_{5}}$ $(c)=z+1$. Partition $E\left(T_{5}\right)$ into 5 sets $E_{1}, E_{2}, E_{3}, E_{4}$, and $E_{5}$ such that $E_{1}=\left\{u v \mid d_{T_{5}}(u)=1\right.$ and $\left.d_{T_{5}}(v)=x+1\right\}, E_{2}=\left\{u v \mid d_{T_{5}}(u)=1\right.$ and $\left.d_{T_{5}}(v)=y+2\right\}, E_{3}=\left\{u v \mid d_{T_{5}}(u)=1\right.$ and $\left.d_{T_{5}}(v)=z+1\right\}$, $E_{4}=\{a b\}, E_{5}=\{b c\}$. It is easy to see that $\left|E_{1}\right|=x,\left|E_{2}\right|=y$, $E_{3}|=z,| E_{4}|=| E_{5} \mid=1$.

Therefore,

$$
\begin{aligned}
& R\left(T_{5}\right)=\sum_{u v \in E\left(T_{5}\right)} \frac{1}{\sqrt{d_{G}(u) d_{G}(v)}} \\
& =\sum_{u v \in E_{1}\left(T_{5}\right)} \frac{1}{\sqrt{d_{G}(u) d_{G}(v)}}+\sum_{u v \in E_{2}\left(T_{5}\right)} \frac{1}{\sqrt{d_{G}(u) d_{G}(v)}}+\sum_{u v \in E_{3}\left(T_{5}\right)} \frac{1}{\sqrt{d_{G}(u) d_{G}(v)}} \\
& +\sum_{u v \in E_{4}\left(T_{5}\right)} \frac{1}{\sqrt{d_{G}(u) d_{G}(v)}}+\sum_{u v \in E_{5}\left(T_{5}\right)} \frac{1}{\sqrt{d_{G}(u) d_{G}(v)}} \\
& =\sum_{u v \in E_{1}\left(T_{5}\right)} \frac{1}{\sqrt{(x+1)(1)}}+\sum_{u v \in E_{2}\left(T_{5}\right)} \frac{1}{\sqrt{(y+2)(1)}}+\sum_{u v \in E_{3}\left(T_{5}\right)} \frac{1}{\sqrt{(z+1)(1)}} \\
& ++\sum_{u v \in E_{4}\left(T_{5}\right)} \frac{1}{\sqrt{(x+1)(y+2)}}+\sum_{u v \in E_{5}\left(T_{5}\right)} \frac{1}{\sqrt{(y+2)(z+1)}} \\
& =\frac{x}{\sqrt{x+1}}+\frac{y}{\sqrt{y+2}}+\frac{z}{\sqrt{z+1}}+\frac{1}{\sqrt{(x+1)(y+2)}}+\frac{1}{\sqrt{(y+2)(z+1)}}
\end{aligned}
$$

Here we have $n=x+y+z+3$. By replacing $z=x+y+z+3$, the above equation reduces to

$$
\begin{aligned}
& R\left(T_{5}\right)=\frac{x}{\sqrt{x+1}}+\frac{y}{\sqrt{y+2}}+\frac{n-x-y-3}{\sqrt{n-x-y-2}}+\frac{1}{\sqrt{(x+1)(y+2)}} \\
& +\frac{1}{\sqrt{(y+2)(n-x-y-2)}} .
\end{aligned}
$$

Proposition 2.4: If $T_{i}$ is a tree with $n$ vertices as shown in Fig. $i$, then the Randić index of $T_{i}, i=6,7,8$ is as follows,

$$
\begin{aligned}
& R\left(T_{6}\right)=\frac{x}{\sqrt{x+1}}+\frac{y}{\sqrt{y+1}}+\frac{n-x-y-4}{\sqrt{n-x-y-3}}+\frac{1}{\sqrt{3(x+1)}}+\frac{1}{\sqrt{3(y+1)}} \\
& +\frac{1}{\sqrt{3(n-x-y-3)}}
\end{aligned}
$$$$
\begin{aligned}
& R\left(T_{7}\right)=\frac{x}{\sqrt{x+1}}+\frac{y}{\sqrt{y+2}}+\frac{n-x-y-5}{\sqrt{n-x-y-4}}+\frac{1}{\sqrt{2(x+1)}}+\frac{2}{\sqrt{2(y+2)}} \\
& +\frac{1}{\sqrt{2(n-x-y-4)}}
\end{aligned}
$$$$
R\left(T_{8}\right)=\frac{x}{\sqrt{x+1}}+\frac{y}{\sqrt{y+2}}+\frac{n-x-y-4}{\sqrt{n-x-y-3}}+\frac{1}{\sqrt{(x+1)(y+2)}}+\frac{1}{\sqrt{2(y+2)}}
$$$$
+\frac{1}{\sqrt{2(n-x-y-3)}}
$$ 


\section{RESULTS FOR COMPLEMENTS}

The complement of a graph $G$, denoted by $\bar{G}$ is a graph with vertex set $V(G)$ and two vertices in $\bar{G}$ are adjacent if and only if they are not adjacent in $G$ [3].

Preposition 2.5: If $G=T_{l}$ is a tree with $n$ vertices and $m$ edges as shown in Fig.1, then the Randić index of complement of $T_{l}$ is

$$
R\left(\bar{T}_{1}\right)=R(\bar{G})=\frac{n-3}{2}+\frac{n-x-2}{\sqrt{(n-2)(n-x-2)}}+\frac{x}{\sqrt{x(n-2)}}
$$

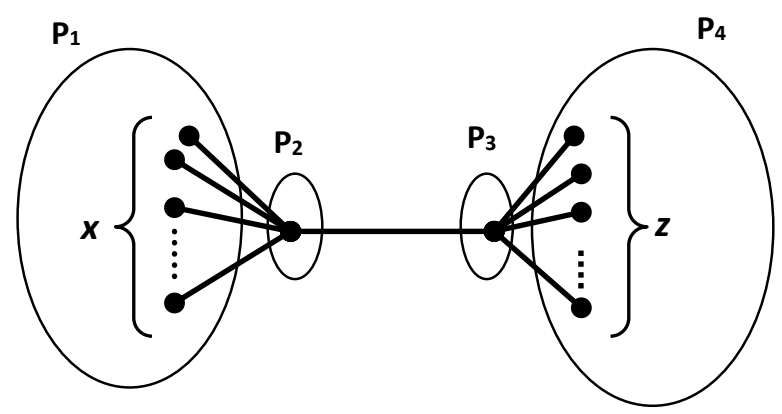

Proof: Consider the partition $\mathbf{P}_{\mathbf{1}}, \mathbf{P}_{\mathbf{2}}, \mathbf{P}_{\mathbf{3}}$ and $\mathbf{P}_{\mathbf{4}}$ ofvertex set of $T_{1}$ as shown in Fig.9. Easily we can note that $\left|\boldsymbol{P}_{1}\right|=\boldsymbol{x},\left|\boldsymbol{P}_{2}\right|=\mathbf{1}$, $P_{3}|=1,| P_{4} \mid=n-x-2$. Therefore,

$$
\begin{aligned}
& R\left(\bar{T}_{1}\right) \quad=\sum_{u v \in E\left(T_{1}\right)} \frac{1}{\sqrt{d_{\bar{G}}(u) d_{\bar{G}}(v)}} \\
& =\sum_{u v \in\left(P_{1}\right)} \frac{1}{\sqrt{d_{\bar{G}}(u) d_{\bar{G}}(v)}}+\sum_{\substack{u \in\left(P_{1}\right) \\
v \in\left(P_{3}\right)}} \frac{1}{\sqrt{d_{\bar{G}}(u) d_{\bar{G}}(v)}}+ \\
& \sum_{\substack{u \in\left(P_{1}\right) \\
v \in\left(P_{4}\right)}} \frac{1}{\sqrt{d_{\bar{G}}(u) d_{\bar{G}}(v)}}+\sum_{\substack{u \in\left(P_{2}\right) \\
v \in\left(P_{4}\right)}} \frac{1}{\sqrt{d_{\bar{G}}(u) d_{\bar{G}}(v)}}+\sum_{u v \in\left(P_{4}\right)} \frac{1}{\sqrt{d_{\bar{G}}(u) d_{\bar{G}}(v)}} \\
& =\sum_{u v \in\left(P_{1}\right)} \frac{1}{\sqrt{(n-1-1)(n-1-1)}}+\sum_{\substack{u \in\left(P_{1}\right) \\
v \in\left(P_{3}\right)}} \frac{1}{\sqrt{(n-1-1)(n-1-(n-x-1))}} \\
& +\sum_{\substack{u \in\left(P_{1}\right) \\
v \in\left(P_{4}\right)}} \frac{1}{\sqrt{(n-1-1)(n-1-1)}}+\sum_{\substack{u \in\left(P_{2}\right) \\
v \in\left(P_{4}\right)}} \frac{1}{\sqrt{(n-1-(x+1))(n-1-1)}} \\
& +\sum_{u v \in\left(P_{4}\right)} \frac{1}{\sqrt{(n-1-1)(n-1-1)}}
\end{aligned}
$$

$$
\begin{aligned}
& =\sum_{u v \in\left(P_{1}\right)} \frac{1}{(n-2)}+\sum_{\substack{u \in\left(P_{1}\right) \\
v \in\left(P_{3}\right)}} \frac{1}{\sqrt{(n-2) x}}+\sum_{\substack{u \in\left(P_{1}\right) \\
v \in\left(P_{4}\right)}} \frac{1}{(n-2)} \\
& +\sum_{\substack{u \in\left(P_{2}\right) \\
v \in\left(P_{4}\right)}} \frac{1}{\sqrt{(n-x-2)(n-2)}}+\sum_{u v \in\left(P_{4}\right)} \frac{1}{(n-2)} \\
& =\frac{n(n-1)}{2(n-2)}+\frac{x}{\sqrt{x(n-2)}}+\frac{x(n-x-2)}{(n-2)}+\frac{n-x-2}{\sqrt{(n-x-2)(n-2)}} \\
& +\frac{(n-x-2)(n-x-3)}{(n-2)} \\
& R\left(\bar{T}_{1}\right)=\frac{n-3}{2}+\frac{n-x-2}{\sqrt{(n-2)(n-x-2)}}+\frac{x}{\sqrt{x(n-2)}}
\end{aligned}
$$

Proposition 2.6: If $T_{i}$ is a tree with $n$ vertices as shown in Fig. $i, i=2,3,4,5$ then the Randic index of complement of $T_{i}$ is as follows,

$$
\begin{aligned}
& R\left(\bar{T}_{2}\right)=\frac{n^{2}-7 n+12}{2(n-2)}+\frac{x}{\sqrt{(n-2)(n-3)}}+\frac{x}{\sqrt{(n-2)(x-3)}}+\frac{x}{\sqrt{(n-2)(x+1)}} \\
& +\frac{1}{\sqrt{(n-x-2)(x+1)}}+\frac{n-x-3}{\sqrt{(n-x-2)(n-2)}}+\frac{n-x-3}{\sqrt{(n-3)(n-2)}}
\end{aligned}
$$$$
R\left(\bar{T}_{3}\right)=\frac{n^{2}-9 n+20}{2(n-2)}+\frac{2 x}{\sqrt{(n-2)(n-3)}}+\frac{x}{\sqrt{(n-2)(x+2)}}
$$$$
+\frac{1}{\sqrt{(n-x-2)(n-3)}}+\frac{1}{\sqrt{(n-x-2)(x+2)}}+\frac{n-x-4}{\sqrt{(n-x-2)(n-2)}}
$$$$
+\frac{1}{\sqrt{(n-3)(x+2)}}+\frac{2(n-x-4)}{\sqrt{(n-3)(n-2)}} \text {. }
$$

$$
R\left(\bar{T}_{4}\right)=\frac{n^{2}-11 n+30}{2(n-2)}+\frac{3 x}{\sqrt{(n-2)(n-3)}}+\frac{x}{\sqrt{(n-2)(x+3)}}
$$$$
+\frac{2}{\sqrt{(n-x-2)(n-3)}}+\frac{1}{\sqrt{(n-x-2)(x+3)}}+\frac{n-x-5}{\sqrt{(n-x-2)(n-2)}}
$$$$
+\frac{1}{n-3}+\frac{2}{\sqrt{(n-3)(x+3)}}+\frac{3(n-x-5)}{\sqrt{(n-3)(n-2)}} \text {. }
$$

$$
\begin{aligned}
& R\left(\bar{T}_{5}\right)=\frac{x(x-1)}{2(n-2)}+\frac{x}{\sqrt{(n-2)(n-z-2)}}+\frac{x}{\sqrt{(n-2)(n-y-3)}}+\frac{x y}{n-2} \\
& +\frac{x z}{n-2}+\frac{y}{\sqrt{(n-x-2)(n-2)}}+\frac{1}{\sqrt{(n-x-2)(n-z-2)}}+\frac{z}{\sqrt{(n-x-2)(n-2)}} \\
& +\frac{y(y-1)}{2(n-2)}+\frac{y}{\sqrt{(n-z-2)(n-2)}}+\frac{y z}{n-2}+\frac{z}{\sqrt{(n-y-3)(n-2)}}+\frac{z(z-1)}{2(n-2)}
\end{aligned}
$$




\section{ALGORITHM:}

$>$ An algorithm to find the Randić index of a graph.

\section{Step 1: START}

Step 2: Declare: $a[25][25], d[25], \mathrm{m}$ as integers sum1,s[25],sum, $t s=0$ as floating points.

Step 3: Read $m, a[i][j]$.

Step 4: Compute : Degree of each vertex of given graph

$$
\begin{aligned}
& \text { for } i \text { to } n \\
& d[i] \leftarrow \quad 0 \\
& \text { for } j \text { to } n \\
& d[i] \leftarrow \quad d[i]+a[i][j]
\end{aligned}
$$

Display: Degree $d[i]$ of vertex $i$

Step 5: Check the condition, if $a[i][j]=1$ is true

$$
\text { Display: Vertex } i \text { is adjacent to vertex } j
$$

Step 6 : Multiply corresponding degrees of adjacent vertices

$$
\operatorname{sum} \leftarrow d[i]^{*} d[j]
$$

Step 6:Display the sum of multiples of adjacent vertices degree

$$
t s \leftarrow t s+(1 / \sqrt{\text { sum }})
$$

Step 7:Display the Randić index by dividing total sum $t s$ by 2 .

\section{Step 8:STOP}

Illustartion:

\section{G:}

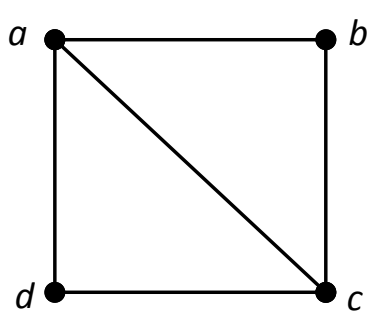

Fig. 10

We represent the graph $\mathrm{G}$ by adjacency matrix,

$$
A(G)=\begin{gathered}
a \\
a \\
b \\
c \\
d
\end{gathered}\left[\begin{array}{llll}
0 & 1 & 1 & 1 \\
1 & 0 & 1 & 0 \\
1 & 1 & 0 & 1 \\
1 & 0 & 1 & 0
\end{array}\right]
$$

In this matrix $a, b, c, d$ represents the vertices of graph $G$. The element 1 in $A(G)$ represents the adjacency between the vertices and $O$ represents the non-adjacency between the vertices. Addition of elements of each row gives the degree of a corresponding vertex in $G$, ie., we get 3 by adding all the elements of a first row of adjacency matrix $A(G)$ which is degree of vertex ' $a$ ' in graph G. similarly we get other vertex degrees by adding corresponding row. Using this we calculate degree of each vertex and store it in $d[i]$ by using for loop.

The outer loop iterates $i$ times and the inner loop iterates $j$ times, the statements inside the inner loop will be executed a total of $i * j$ times. It is because, inner loop will iterate $j$ times for each of the $i$ iterations of the outer loop. This means the outer and inner loop are dependent on the problem size ie., here we considered size is $n$, the statement in the whole loop will be executed $O\left(n^{2}\right)$ times. In the loop int $i=0$, this will be executed only once. The time is actually calculated to $i=0$ and not the declaration, $i<n$ this will be executed $n+1$ times, $i++$ will be executed $n$ times, $a[i][j]=1$, This will be executed $n$ times (in worst case scenario).

As per the definition Randic index we multiply the degree of vertices which are adjacent, by adjacency matrix $A(G)$, we check the adjacency of one vertex to another by using if condition, then we multiply the degree of those adjacency vertices using $d[i]$,(This loop follows same procedure as explained for above loop so this also executed $\mathrm{O}\left(n^{2}\right)$ times). Then we sum the multiplied valueof each adjacent vertices and each time we store the resulting value in one variable say $t s$ as per the definition of Randic index,ie., $1 / \sqrt{s u m}$. So for the above example we get final value of $t s$ as 3.9266 . We obtain the Randić index by dividing $t s$ by 2 .Therefore the Randić index of this example is 1.9633 .

\section{CONCLUSION}

The results give explicit formulas for Randic index of certain class of trees and also for their complements. Further an algorithm with the help of adjacency matrix given to compute the Randić index of graph. 


\section{ACKNOWLEDGEMENTS}

This work is supported by the University Grants Commission (UGC), Govt. of India for support through research grant under UPE FAR-II grant No. F14-3/2012 (NS/PE).

\section{REFERENCES :}

1. I. Gutman, B. Furtula (Eds.), Recent Results in the Theory of Randic' Index,Uni. Kragujevac, Kragujevac, 2008.

2. X. Li, I. Gutman, Mathematical Aspects of Randic'-Type Molecular Structure Descriptors, Uni. Kragujevac, Kragujevac, 2006.

3. F. Harary, Graph Theory, Addison-Wesley, Reading, MA, 1969.

4. X. Li, Y. Shi, A survey on the Randic index, MATCH Commun. Math. Comput. Chem., 59 (2008),127-156.

5. H. S. Ramane, B. Basavanagoud, R. B. Jummannaver, Harmonic index and Randić index of generalized transformation graphs, preprint.

6. H. S. Ramane, R. B. Jummannaver, S. C. Patil, "Harmonic index of some class of trees with an algorithm", IOSR Journal of Mathematics (IOSR-JM).12(2) (2016), 52-57

7. M. Randić, On characterization of molecular branching, J. Am. Chem. Soc. 97 (1975) 6609-6615.

8. M. Randić, On history of the Randi'c index and emerging hostility toward chemical graph theory.MATCH Commun. Math. Comput. Chem. 59(2008), 5-124 .

9. M. Randi'c, The connectivity index 25 years later. J. Mol. Graph. Model. 20(2001), 19-35. 